\title{
Protection et droits liés à la grossesse et à la maternité en droit de l'UE
}

Susanne Burri

\section{(2) OpenEdition}

1 Journals

\section{Édition électronique}

URL : https://journals.openedition.org/rdctss/1654

DOI : $10.4000 /$ rdctss. 1654

ISSN : 2262-9815

Éditeur

Centre de droit comparé du travail et de la sécurité sociale

\section{Édition imprimée}

Date de publication : 1 avril 2019

Pagination : 88-97

ISSN : 2117-4350

\section{Référence électronique}

Susanne Burri, «Protection et droits liés à la grossesse et à la maternité en droit de l'UE », Revue de droit comparé du travail et de la sécurité sociale [En ligne], 1 | 2019, mis en ligne le 01 novembre 2021, consulté le 13 novembre 2021. URL : http://journals.openedition.org/rdctss/1654 ; DOI : https:// doi.org/10.4000/rdctss. 1654

\section{(c) $(7)(9$}

Revue de droit comparé du travail et de la sécurité sociale est mise à disposition selon les termes de la Licence Creative Commons Attribution - Pas d'Utilisation Commerciale - Pas de Modification 4.0 International. 


\title{
PROTECTION ET DROITS LIÉS À LA GROSSESSE ET À LA MATERNITÉ EN DROIT DE L'UE
}

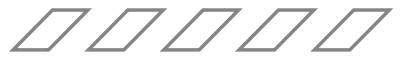

\section{RÉSUMÉ}

La Cour de Justice a dès 1990 fermement établi le principe de non-discrimination fondée sur le sexe en relation aux femmes enceintes dans l'accès à l'emploi, les conditions de travail et le licenciement. Des obligations spécifiques reposent sur les États membres et les employeurs depuis l'adoption de la directive 92/85, qui n'a pas été amendée depuis. La jurisprudence de la Cour a considérablement renforcé la protection contre un refus d'embauche ou un licenciement liés à la grossesse. Cet article propose un court aperçu de la législation de l'UE dans ce domaine et une analyse de la jurisprudence de la Cour sur la protection et les droits liée à la grossesse et la maternité.

MOTS CLÉS : Discriminations fondées sur le sexe, grossesse, maternité, droit de l'UE, jurisprudence de la CJUE.

\begin{abstract}
The European Court of Justice has since 1990 firmly established the principle of non-discrimination on the ground of sex in relation to pregnant women in the access to employment, working conditions and dismissal. Specific obligations apply to member states and employers since directive 92/85 entered into force, which has not been amended since. The case of the Court has significantly enhanced the protection against a refusal to enroll or a dismissal linked to pregnancy. This contribution offers a short overview of the relevant EU legislation and an analysis of the case law of the Court on the protection and rights in relation to pregnancy and maternity.
\end{abstract}

KEYWORDS : Sex Discrimination, Pregnancy, Maternity, EU Law, CJEU Case Law. 


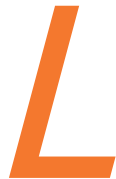

a jurisprudence de la Cour de Justice concernant l'interdiction de discrimination directe fondée sur le sexe a depuis 1990 contribué à protéger les femmes participant au marché du travail en particulier contre un refus d'embauche, des conditions de travail désavantageuses et des licenciements liés à la grossesse

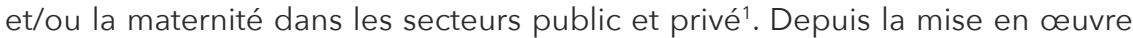
de la directive 92/85 visant à promouvoir l'amélioration de la sécurité et de la santé des travailleuses enceintes, accouchées ou allaitantes, des protections et droits spécifiques existent en plus dans ce domaine ${ }^{2}$. Une modification de cette directive a été proposée en 2008, incluant entre autre un droit à un congé de maternité de 18 semaines entièrement rémunéré au lieu des 14 semaines existantes à un salaire réduit ${ }^{3}$. De longues négociations n'ayant pas abouti, la Commission a retiré cette proposition de directive en juillet $2015^{4}$. En relation à la grossesse, l'accouchement et l'allaitement, c'est donc toujours la directive de 1992 qui indique le minimum de protections et de droits requis au niveau national. La Cour de Justice a souvent été invitée à interpréter le droit de l'UE en réponse à des questions préjudicielles de juges nationaux des États membres en relation à la grossesse et la maternité. Certains aspects de la jurisprudence de la Cour ont été incorporés dans la directive refonte 2006/54 - qui a remplacé la directive 76/207 - relative à la mise en œuvre du principe de l'égalité des chances et de l'égalité de traitement entre hommes et femmes en matière d'emploi et de travail 5 .

Parallèlement, la conciliation de la vie professionnelle, familiale et privée fit l'objet d'une directive mettant en œuvre un accord entre les partenaires sociaux européens sur

1 Cette jurisprudence concerne en premier lieu l'interprétation de l'article 119 CEE (141 CE, 157 TFUE) sur l'égalité de rémunération entre travailleurs masculins et féminins et la Directive 76/207/ CEE du Conseil, du 9 février 1976, relative à la mise en œuvre du principe de l'égalité de traitement entre hommes et femmes en ce qui concerne l'accès à l'emploi, à la formation et à la promotion professionnelles, et les conditions de travail, JO L 039 du 14.02.1976, p. 40.

2 Directive 92/85/CEE du Conseil, du 19 octobre 1992, concernant la mise en œuvre de mesures visant à promouvoir l'amélioration de la sécurité et de la santé des travailleuses enceintes, accouchées ou allaitantes au travail (dixième directive particulière au sens de l'article 16 paragraphe 1 de la directive 89/391/CEE), JO L 348, 28.11.1992, p. 1.

$3 \operatorname{COM}(2008) 637$.

4 Commission européenne, - Communiqué de presse, Faciliter la vie des parents: la Commission retire sa proposition en attente sur le congé de maternité et ouvre la voie à une nouvelle approche,

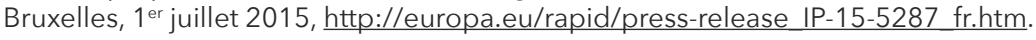

5 Directive 2006/54/CE du Parlement européen et du Conseil du 5 juillet 2006 relative à la mise en œuvre du principe de l'égalité des chances et de l'égalité de traitement entre hommes et femmes en matière d'emploi et de travail (refonte), JO L 204 du 26.7.2006, p. 23. 
le congé parental en $1996 .{ }^{6}$ Cette directive, remplacée par la Directive 2010/187 sera probablement de nouveau remplacée dans un proche avenir. En effet, en janvier 2019, un accord provisoire a été conclu entre le Parlement européen et le Conseil sur la proposition de directive de la Commission concernant l'équilibre entre la vie professionnelle et vie privée des parents et des aidants ${ }^{8}$.

La présente contribution propose une analyse de la jurisprudence de la Cour sur la protection des femmes liée à la grossesse et la maternité, ainsi que les droits spécifiques des femmes salariées liés à la naissance de leur(s) enfant(s). On donnera d'abord un aperçu de la législation de I'UE dans ce domaine (I) ${ }^{9}$. Ces normes garantissent une protection minimale; les États membres sont libres d'adopter des dispositions plus favorables pour les travailleurs. Mais l'interprétation de la Cour de Justice doit être prise en compte au niveau national par le législateur, les juges, les organes de mise en œuvre du droit de l'UE, les partenaires sociaux et les employeurs. Il est donc utile de rappeler ces obligations, ainsi que leur champ d'application. Le but de cette contribution est de présenter les normes minimales les plus importantes dans ce domaine sans toutefois commenter en détail tous les arrêts de la Cour de Justice de l'UE pertinents ${ }^{10}$.

\section{I - LA LÉGISLATION}

L'article 157 TFUE consacre le principe de l'égalité des rémunérations entre hommes et femmes et peut être invoqué au niveau national non seulement dans des litiges entre (un organe de) l'Etat, mais également entre particuliers ${ }^{11}$. Cet article interdit les discriminations

6 Directive 96/34/CE du Conseil du 3 juin 1996 concernant l'accord-cadre sur le congé parental conclu par I'UNICE, le CEEP et la CES, JO L 145 du 19.6.1996, p. 4.

7 Directive 2010/18/UE du Conseil du 8 mars 2010 portant application de l'accord-cadre révisé sur le congé parental conclu par BUSINESSEUROPE, I'UEAPME, le CEEP et la CES et abrogeant la directive 96/34/CE (Texte présentant de l'intérêt pour l'EEE), JO L 68 du 18.3.2010, p. 13.

Voir S. Burri, « La conciliation de la vie professionnelle, privée et familiale. L'approche juridique de I'Union européenne », Revue de l'OFCE, 2010, n 114, p. 213.

8 Commission européenne, déclaration, 24 janvier 2019, http://europa.eu/rapid/press-release STATEMENT-19-424 fr.htm et Proposition de Directive du Parlement européen et du Conseil concernant l'équilibre entre vie professionnelle et vie privée des parents et aidants et abrogeant la directive 2010/18/UE du Conseil, Bruxelles, le 26.4.2017 COM(2017) 253 final 2017/0085 (COD), https://eur-lex.europa.eu/resource.html?uri=cellar:84205176-2b39-11e7-941201aa75ed71a1.0003.02/DOC 1\&format=PDF. Voir S. Burri, "The EU Proposal on Work-Life Balance and its Potential Impact in the Netherlands», in J. Pichrt, Kristina Koldinská (dir.), Labour Law and Social Protection in a Globalized World - Changing Realities in Selected Areas of Law and Policy, Alphen aan den Rijn, Kluwer Law International, 2018, p. 305.

9 Cette contribution ne concerne ni la sécurité sociale (Directive 79/7), ni les travailleurs indépendants (Directive 2010/41), ni les services (Directive 2004/113). Voir pour un aperçu général, S. Burri, EU Gender equality law - update 2018, European network of legal experts in gender equality and nondiscrimination, European Commission, novembre 2018, https://www.equalitylaw.eu/downloads/4767-eu-gender-equality-law-update-2018-pdf-444-kb.

10 Jusqu'au $1^{\text {er février } 2019 .}$

11 Depuis le 8 avril 1976 (CJUE 8 avril 1976, C-43/75, (Defrenne II), ECLI:EU:C:1976:56). Voir aussi I'article 4 de la Directive 2006/54. 
directes fondées sur le sexe ${ }^{12}$ - par exemple un désavantage lié à la grossesse ou à un congé de maternité ${ }^{13}$ - et les discriminations indirectes. Ceci pourrait par exemple être le cas si beaucoup plus de femmes que d'hommes en congé parental subissaient des désavantages liés à ce congé suite à un critère, une mesure ou une pratique neutre par rapport au sexe. Une telle mesure est interdite si elle ne peut être objectivement justifiée par un but légitime et des moyens appropriés et nécessaires pour atteindre ce but ${ }^{14}$.

La directive 2006/54 interdit les discriminations fondées sur le sexe en particulier dans l'accès à l'emploi, la formation professionnelle, la promotion, les conditions d'emploi ainsi que le licenciement (article 14). L'interdiction porte sur les discriminations directes et indirectes ainsi que sur le harcèlement lié au sexe et le harcèlement sexuel, concepts qui sont définis dans la directive (article $2: 1$ ). L'injonction de pratiquer à l'encontre de personnes une telle discrimination est également interdite (article $2: 2 \mathrm{~b}$ ). Ceci pourrait par exemple être le cas d'un employeur exigeant d'une agence temporaire de ne pas proposer une femme enceinte pour remplir temporairement certaines tâches administratives qu'elle serait apte à remplir. Explicitement proscrit est tout traitement moins favorable d'une femme lié à la grossesse ou au congé de maternité dans le sens de la directive 92/85 (article 2 :2c). Une femme en congé de maternité a le droit, au terme de ce congé, de retrouver son emploi ou un emploi équivalent à des conditions qui ne lui soient pas moins favorables et de bénéficier de toute amélioration des conditions de travail à laquelle elle aurait eu droit durant son absence (article 15). Des dispositions protectrices en relation à la grossesse et la maternité sont permises (article 28).

Des droits et obligations spécifiques liés à la grossesse et la maternité découlent de la directive 92/85. Cette directive s'applique aux travailleuses enceintes, accouchées ou allaitantes (article 2$)^{15}$. La directive contient des lignes directrices pour protéger la santé de ces travailleuses. Ainsi, il est interdit de les exposer à des agents chimiques ou biologiques dangereux (articles 3-6) et le travail de nuit ${ }^{16}$ est interdit pendant la grossesse et une certaine période après l'accouchement (article 7). La Cour a dans deux arrêts jugé que l'article 19 de la directive 2006/54 sur la charge de la preuve s'applique à une situation dans laquelle une travailleuse allaitante conteste une décision relative à une évaluation des risques (exigée par l'article $4: 1$ de la directive 92/85) devant une juridiction nationale ou toute autre instance compétente de l'État ${ }^{17}$. Le congé de maternité doit comprendre 14

12 Voir la définition de discrimination directe par exemple dans l'article 2:1 a de la directive 2006/54.

13 Comme dans le cas d'une prime de Noël, lorsque l'employeur réduit proportionnellement la prestation en tenant compte de périodes de protection de la mère qui lui interdisent de travailler : arrêt du 21 octobre 1999, C-333/97 (Lewen), ECLI:EU:C:1999:512 (point 51).

14 Voir la définition de discrimination indirecte par exemple dans l'article $2: 1 \mathrm{~b}$ de la directive 2006/54.

15 Voir sur la notion de travailleuse enceinte en particulier: l'arrêt du 26 février 2008, C-506/06, (Mayr), ECLI:EU:C:2008:119 et l'arrêt du 11 novembre 2010, C-232/09, (Danosa), ECLI :EU:C:2010:674.

16 Voir par exemple sur l'interprétation de la Cour des dispositions de la directive 76/207 (maintenant abrogée et remplacée par la Directive 2006/54) et le travail de nuit : arrêts de la Cour du 25 juillet 1991, C-345/89, (Stoeckel), ECLI:EU:C:1991:324; 3 février 1995, C-13/93, (Minne), ECLI:EU:C:1994:39; 5 mai 1994, C-421/92, (Habermann-Beltermann), ECLI:EU:C:1994:187; 13 mars 1997, C-197/96, (Commission/France), ECLI:EU:C:1997:155; 4 décembre 1997, C-207/96, (Commission/ltalie), ECLI:EU:C:1997:583 et 19 septembre 2018, C-41/17, (González Castro), ECLI:EU:C:2018:736.

17 Arrêts du 19 octobre 2017, C-531/15, (Otero Ramos), ECLI:EU:C:2017:789 et 19 septembre 2018, C-41/17, (González Castro), ECLI:EU:C:2018:736. 
semaines continues au moins, réparties avant et/ou après l'accouchement, dont au moins 2 semaines de congé de maternité obligatoire (article 8$)^{18}$. Durant ce congé, il existe un droit à une rémunération ou une prestation adéquate, qui doit être au moins équivalente à la prestation qu'une travailleuse recevrait en cas d'interruption de ses activités pour des raisons liées à son état de santé (art. 11) ${ }^{19}$. L'article 10 interdit le licenciement depuis le début de la grossesse jusqu'à la fin du congé de maternité (dans le sens de l'article 8), sauf en cas d'exception non liés à la grossesse ou la maternité ${ }^{20}$. Cet article a un effet direct ${ }^{21}$.

La Chartre des droits fondamentaux de I'UE est également pertinente en relation aux principes d'égalité, de non-discrimination et d'égalité entre hommes et femmes (articles 20, 21 et 23). De plus, l'article 33 sur la vie familiale et professionnelle stipule :

1 - La protection de la famille est assurée sur le plan juridique, économique et social.

2 - Afin de pouvoir concilier vie familiale et vie professionnelle, toute personne a le droit d'être protégée contre tout licenciement pour un motif lié à la maternité, ainsi que le droit à un congé de maternité payé et à un congé parental à la suite de la naissance ou de l'adoption d'un enfant.

La Cour a reconnu que cet article reflète un droit social fondamental22. Cet article pourrait à l'avenir jouer un rôle plus pertinent dans des relations horizontales (par exemple entre salarié et employeur). La Cour a en effet récemment dans l'arrêt Bauer explicitement reconnu l'invocabilité de l'article 31:2 de la Charte sur le congé annuel dans le cadre d'un litige entre particuliers lorsqu'il est impossible d'interpréter le droit national conformément à une disposition d'une directive ${ }^{23}$.

\section{II - LA JURISPRUDENCE DE LA COUR DE JUSTICE}

Déjà en 1996, la Cour a reconnu que les femmes qui bénéficient d'un congé de maternité prévu par la législation nationale se trouvent dans une 'situation spécifique qui exige qu'une protection spéciale leur soit accordée, mais qui ne peut pas être assimilée à celle d'un homme ni à celle d'une femme qui occupe effectivement son poste de travail'24.

18 Voir par exemple l'arrêt du 27 octobre 1998, C-411/96, (Boyle), ECLI :EU:C:1998:506, points 45-54 et 55-66.

19 Voir par exemple l'arrêt du 27 octobre 1998, C-411/96, (Boyle), ECLI:EU:C:1998:506, points 28-44 et 81-87.

20 Voir aussi l'arrêt du 30 juin 1998, C-394/96 (Brown), ECLI:EU:C:1998:331.

21 Arrêt du 4 octobre 2001, C-438/99, (Jiménez Melgar), ECLI:EU:C:2001:509, points 31-34.

22 Arrêt du 16 septembre 2010, C-149/10, (Zoi Chatzi), ECLI:EU:C:2010:534, point 63.

23 Arrêt du 6 novembre 2018, affaires jointes C-569/16 et C-570/16, (Bauer), ECLI:EU:C:2018:871, points 64-92. Voir sur l'applicabilité de l'article 21 de la Charte (égalité de traitement) également l'arrêt du 22 janvier 2019, C-193/17, (Cresco Investigation), ECLI:EU:C:2019:43 et la jurisprudence citée dans cet arrêt.

24 Arrêt du 13 février 1996, C-342/93 (Gillespie), ECLI:EU:C:1999:46, point 17. 


\section{A - L'ACCÈs À L'EMPLOI}

Dans le fameux arrêt Dekker la Cour a jugé que refuser un emploi à une femme du fait de sa grossesse est une discrimination directe fondée sur le sexe et que seules les exceptions prévues par la législation peuvent être invoquées ${ }^{25}$. Dans ce cas, une comparaison avec un candidat masculin n'est pas requise ${ }^{26}$. Dans l'arrêt Jiménez Melgar la Cour a jugé que dans la mesure où le non-renouvellement d'un contrat de travail à durée déterminée est motivé par l'état de grossesse d'une travailleuse, il constitue une discrimination directe fondée sur le sexe ${ }^{27}$. Cette protection est d'importance vu le nombre important de cdd. Ainsi, en ce qui concerne l'accès à l'emploi, la jurisprudence de la Cour est stricte. Ceci est moins le cas pour la rémunération.

\section{B - LA RÉMUNÉRATION}

Lorsque la rémunération versée pendant le congé de maternité constitue une rémunération dans le sens de l'article 119 CEE (141 CE, 157 TFUE) et de la directive 75/11728 (maintenant abrogée par la directive 2006/54), cet article n'impose pas l'obligation de maintenir la rémunération intégrale des travailleuses pendant leur congé de maternitéz ${ }^{2}$. En cas d'état pathologique lié à la grossesse, les salariées incapables de travailler pour cette raison bénéficient en principe du même salaire que les autres salariés inaptes au travail pour cause de maladie ${ }^{30}$. Mais une législation nationale qui prévoit qu'un employeur peut, lorsqu'il estime ne pouvoir employer une femme enceinte qui n'est pourtant pas inapte au travail, la renvoyer chez elle sans lui payer l'intégralité de son salaire, est contraire aux directives $76 / 207$ et $92 / 85^{31}$.

La Cour a jugé qu'un régime de congé de maladie qui traite de manière identique les travailleuses souffrant d'une maladie liée à une grossesse et les autres travailleurs atteints d'une maladie indépendante d'un état de grossesse relève du champ d'application de l'article 141 CE (157 TFUE) et de la directive 75/11732. Le droit de I'UE n'impose pas le maintien intégral de la rémunération d'une salariée absente en raison d'une maladie liée à

25 Voir les articles 14:2 (activités professionnelles particulières); 28 :1 (protection de la femme, notamment en ce qui concerne la grossesse et la maternité) et 3 (action positive) de la directive 2006/54.

26 Arrêt du 8 novembre 1990, affaire 177/88, (Dekker), ECLI:EU:C:1990:383, points 15-18. Voir également par exemple l'arrêt du 3 février 2000, C-207/98 (Mahlburg), ECLI:EU:C:2000:64 et l'article 2 :2c de la directive 2006/54.

27 Arrêt du 4 octobre 2001, C-438/99, (Jiménez Melgar), ECLI:EU:C:2001:509. Suivant les circonstances, il s'agit d'un refus d'embauche : points 43-47.

28 Directive 75/117/CEE du Conseil, du 10 février 1975, concernant le rapprochement des législations des États membres relatives à l'application du principe de l'égalité des rémunérations entre les travailleurs masculins et les travailleurs féminins,Directive 75/117/CEE du Conseil, du 10 février 1975, concernant le rapprochement des législations des États membres relatives à l'application du principe de l'égalité des rémunérations entre les travailleurs masculins et les travailleurs féminins JO L 45, 19.2.1975, p. 19-20.

29 Arrêt du 13 février 1996, C-342/93, (Gillespie), ECLI:EU:C:1999:46, point 25.

30 Arrêt du 19 novembre 1998, C-66/96, (Høj Pedersen), ECLI:EU:C:1998:549.

31 Høj Pedersen, points 51-59.

32 Arrêt du 8 septembre 2005, C-191/03, (McKenna), ECLI:EU:C:2005:513. 
la grossesse pendant la grossesse, à condition qu'elle soit traitée de la même façon qu'un salarié absent pour cause de maladie et que le montant des prestations versées ne soit pas minime au point de mettre en cause l'objectif de protection des travailleuses enceintes. II en est de même pour la rémunération durant des absences pour maladie liée à la grossesse après la fin du congé de maternité.

Depuis l'entrée en vigueur de la directive 92/85, l'article 11 :2b établit le droit au maintien d'une rémunération ou d'une prestation adéquate pendant le congé de maternité ${ }^{3}$. La notion de rémunération dans cet article 'englobe, à l'instar de la définition énoncée à l'article 141, paragraphe 2, premier alinéa, CE, les avantages que l'employeur paie directement ou indirectement pendant le congé de maternité en raison de l'emploi de la travailleuse. En revanche, la notion de prestation à laquelle se réfère également cette disposition comprend tout revenu que la travailleuse perçoit pendant son congé de maternité et qui ne lui est pas versé par son employeur au titre de la relation de travail'34. Cet article a un effet direct ${ }^{35}$. Deux arrêts publiés le même jour concernent le droit à des primes durant la grossesse et le congé de maternité ${ }^{36}$. L'arrêt Gassmayr clarifie qu'une travailleuse enceinte dispensée provisoirement de travailler en raison de sa grossesse a droit au salaire moyen qu'elle recevait avant le début de sa grossesse, mais pas à une indemnité pour astreinte sur lieu de travail. Il en est de même en ce qui concerne la rémunération durant le congé de maternité ${ }^{37}$. La Cour a précisé dans l'arrêt Parviainen qu'une travailleuse qui a été provisoirement affectée, en raison de sa grossesse, sur un poste dans lequel elle effectue des tâches autres que celle qu'elle effectuait antérieurement, a droit non seulement au maintien à son salaire de base, mais également aux primes qui se rattachent à son statut professionnel ${ }^{38}$. Ce sont par exemple des primes relatives à sa qualité de supérieur hiérarchique, à son ancienneté et à ses qualifications professionnelles.

\section{C - CONDITIONS D'EMPLOI ET CONGÉ DE MATERNITÉ}

La jurisprudence de la Cour concernant les conditions d'emploi a surtout trait au congé de maternité et offre différentes sortes de protection. Ainsi, une salariée n'est pas tenue d'informer son employeur qu'elle est enceinte, car l'employeur ne peut pas prendre en considération l'état de grossesse de la salariée pour l'application de ses conditions de travail. Une salariée qui souhaite réintégrer son emploi avant la fin d'un congé parental n'est donc pas obligée d'informer son employeur de son état de grossesse, lorsqu'elle ne pourrait exercer certaines de ses fonctions en raison de certaines interdictions légales ${ }^{39}$.

33 Voir le droit au maintien de la rémunération en relation à un congé parental précédant le congé de maternité par exemple l'arrêt du 13 février 2014, affaires jointes C-512/11 et 513/11, (TSN), ECLI:EU:C:2014:73.

34 Arrêt du 27 octobre 1998, C-411/96, (Boyle), ECLI:EU:C:1998:506, point 31.

35 Arrêt du 1er juillet 2010, C-194/08, (Gassmayr), ECLI: EU:C:2010:386, points 43-53.

36 Voir aussi l'arrêt du 21 octobre 1999, C-333/97 (Lewen), ECLI:EU:C:1999:512.

37 Arrêt du 1er juillet 2010, C-194/08, (Gassmayr), ECLI:EU:C:2010:386, points 54-91.

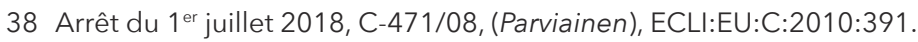

39 Arrêt du 27 février 2003, C-320/01 (Busch), ECLI:EU:C:2003:114, points 38-47. Voir aussi par exemple l'arrêt du 20 septembre 2007, C-116/06 (Kiiski), ECLI:EU:C:2007:536. 
Priver une salariée du droit d'être notée et, par voie de conséquence, de pouvoir profiter d'une promotion professionnelle en raison d'une absence pour congé de maternité est une discrimination directement fondée sur le sexe ${ }^{40}$. Le congé de maternité n'ayant pas été pris en compte pour le calcul de la période requise de six mois de présence en une année pour avoir droit à une notation, ce traitement défavorable est interdit. La Cour a de plus précisé dans l'arrêt Thibault que la directive 76/207 vise à déboucher sur une égalité substantielle et non formelle ${ }^{41}$.

Si le congé de maternité coïncide avec le congé annuel fixé par accord collectif pour l'ensemble du personnel, une salariée doit pouvoir bénéficier de son congé annuel lors d'une période distincte de celle de son congé de maternité. La finalité du droit au congé annuel est différente de celle du droit au congé de maternité ${ }^{42}$. Ce dernier vise, d'une part, à la protection de la condition biologique de la femme au cours de sa grossesse et à la suite de celle-ci et, d'autre part, à la protection des rapports particuliers entre la femme et son enfant au cours de la période qui fait suite à la grossesse et à l'accouchement ${ }^{43}$. Une mère commanditaire ayant eu un enfant grâce à une convention de mère porteuse n'a pas droit au congé de maternité, même si elle allaite son enfant ${ }^{44}$.

\section{D - LICENCIEMENT}

La Cour a déjà en 1990 jugé que le licenciement d'une salariée en raison de sa grossesse constitue une discrimination fondée sur le sexe ${ }^{45}$. Durant le congé de maternité, la femme est protégée contre les licenciements motivés par son absence. Mais en cas d'absences pour cause de maladie liée à la grossesse ou l'accouchement qui apparaît après ce congé, le régime général s'applique ${ }^{46}$. La jurisprudence de la Cour n'offre donc pas de protection spécifique après la fin du congé de maternité dans un tel cas.

40 Arrêt du 30 avril 1998, C-136/95, (Thibault), ECLI:EU:C:1998:178. Voir aussi par exemple l'arrêt du 16 février 2006, C-294/04, (Sarkatzis Herrero), ECLI:EU:C:2006:109.

41 Arrêt du 30 avril 1998, C-136/95, (Thibault), ECLI:EU:C:1998:178, point 26.

42 Arrêt du 18 mars 2004, C-343/01, (Merino Gómez), ECLI:EU:C:2004:160, points 28-41.

43 Arrêt du 12 juillet 1984, 184/83, (Hoffmann), ECLI:EU:C:1984:273, point 25. Voir sur le droit du père à un congé dont bénéficient les mères après l'accouchement : arrêts du 30 septembre 2010, C-104/09, (Roca Álvarez), ECLI:EU:C:2010:561 et du 19 septembre 2013, C-5/12, (Betriu Montull), ECLI:EU:C:2013 :571. Voir sur l'approche de la CJEU dans ces arrêts, E. Caracciolo di Torella, "Brave New Fathers for a Brave New World? Fathers and Caregivers in an evolving European Union », European Law Journa, 2014, vol.20, n\%, p. 88. S. Burri, «Parents who want to reconcile work and care: which equality under EU law ? ", in M. van den Brink, S. Burri, J. Goldschmidt (dir.), Equality and human rights: nothing but trouble? Liber amicorum Titia Loenen, Utrecht: SIM/ Universiteit Utrecht, 2015, p. 261.

44 Arrêts du 18 mars 2014,C-167/12(C.D.), ECLI:EU:C:2014:169 etC-363/12,(Z.), ECLI:EU:C:2014:159. Voir pour une analyse de ces arrêts, S. Burri, "Care in Family Relations - the Case of Surrogacy Leave ", European Journal of Law Reform, 2015, vol.17, n², p. 271. E. Carracciolo di Torella, P. Foubert, «Surrogacy, Pregnancy and Maternity Rights: A Missed Opportunity for a More Coherent Regime of Parental Rights in the EU», European Law Review, 2015, vol.40, n¹0, p. 52.

45 Arrêt du 8 novembre 1990, C-179/88, (Hertz), ECLI:EU:C:1990:384.

46 Hertz, points 17-19. 
Plusieurs arrêts concernent la protection contre le licenciement et des protections visant à renforcer la position des travailleuses confrontées à des licenciements liées à la grossesse et/ou la maternité. L'article 10 de la directive 92/85 interdit le licenciement des travailleuses enceintes, accouchées ou allaitantes depuis le début de leur grossesse jusqu'au terme du congé de maternité. Cet article interdit non seulement de notifier une décision de licenciement en raison de la grossesse et/ou de la naissance d'un enfant pendant la période de protection visée par cet article, mais également de prendre des mesures préparatoires à une telle décision avant l'échéance de cette période ${ }^{47}$. Une telle décision est en outre contraire à la directive 76/207 (maintenant abrogée par la directive 2006/54), quel que soit le moment où elle est notifiée ${ }^{48}$.

Les États membres de I'UE sont obligés d'introduire des mesures nécessaires pour assurer une protection juridictionnelle au travailleur dont l'employeur refuse, après la cessation de la relation de travail, de fournir des références en réaction à une action en justice introduite pour faire respecter le principe d'égalité de traitement ${ }^{49}$. Une voie de recours spécifique relative à l'interdiction du licenciement des travailleuses enceintes, accouchées ou allaitantes n'est pas contraire au droit de l'UE, pour autant que les modalités procédurales propre à ce recours ne soient pas moins favorables que des recours similaires en droit interne. Les principes d'équivalence et d'effectivité doivent être respectés ${ }^{50}$.

La directive 76/207 et l'article 10 de la directive 92/85 interdisent le licenciement lié à la grossesse d'une femme engagée pour une durée déterminée qui ne peut pas travailler pendant une grande partie de la durée de son contrat ${ }^{51}$. La durée du contrat n'a pas d'incidence sur l'étendue de la protection garantie par le droit de I'UE aux travailleuses enceintes. Cette interdiction s'applique également lorsque la travailleuses a omis d'informer son employeur de son état de grossesse. Cette jurisprudence illustre l'ample protection garantie aux femmes enceintes contre un licenciement discriminatoire. Des exceptions non liées à l'état des travailleuses enceintes, accouchées ou allaitantes sont permises seulement sous certaines conditions, par exemple en cas de licenciements collectifs ${ }^{52}$.

47 Arrêt du 11 octobre 2007, C-460/06, (Paquay), ECLI:EU:C:2007:601.

48 Paquay, points 34-54.

49 Arrêt du 22 septembre 1998, C-185/97), (Coote), ECLI:EU:C:1998:424. Dans ce cas, il s'agissait d'une action en justice pour cause de discrimination fondée sur le sexe relative à la grossesse.

50 Arrêt du 29 octobre 2009, C-63/08, (Pontin), ECLI:EU:C:2009:666.

51 Arrêt du 4 octobre 2001, C-109/00, (Tele Danmark), ECLI:EU:C:2001:513. Voir aussi arrêt du 14 juillet 1994, C-32/93, (Webb), ECLI:EU:C:1994:300.

52 Voir en particulier l'arrêt du 22 février 2018, C-103/16, (Porras Guisado), ECLI:EU:C:2018:99. 


\section{DROITS À LA GROSSESSE ET À LA MATERNITÉ DANS L'UE}

\section{Conclusion}

Même si le législateur de I'UE n'a pas réussi à moderniser et amplifier la protection et les droits des travailleuses enceintes, accouchées ou allaitantes, la jurisprudence de la Cour offre une ample protection en particulier contre les discriminations dans l'accès à l'emploi et les licenciements. Cette protection s'étend aux cdd. Des dispositions spécifiques s'appliquent, en plus du principe d'égalité de traitement qui peut jouer un rôle dans les cas où les droits existants ne pourraient être invoqués dans des situations particulières ${ }^{53}$. Quelques aspects de cette jurisprudence ont été codifiés dans la directive 2006/54, mais certainement pas tous. La riche et ample jurisprudence de la Cour en la matière mérite - ne serait-ce que pour cette raison - certainement d'être mieux connue.

53 Voir en relation à la protection contre le licenciement d'une travailleuse soumise à un traitement de fécondation in vitrio : I'arrêt du 26 février 2008, C-506/06, (Mayr), ECLI:EU:C:2008:119, point 44-54 et en relation au congé parental l'arrêt du 16 septembre 2010, C-149/10, (Zoi Chatzi), ECLI:EU:C:2010:534, points 63-75.

\section{SUSANNE BURRI}

Professeure associée de Droit au Département du droit de I'Université d'Utrecht, Pays-Bas.

Thèmes de recherche : Principe d'égalité en droit de l'UE, néerlandais et international, articulation vie professionnelle et privée, relations de travail atypiques, familles et droit.

\section{Publications:}

S. Burri, « Articuler vie professionnelle, familiale et privée aux Pays-Bas - quelle flexibilité pour les salariés ? », Revue de Droit Comparé du Travail et de la Sécurité Sociale, 2018 (1), p. 32.

S. Burri, EU Gender Equality Law. Update 2018, Luxembourg, Publications Office of the European Union, 2018, 54 pages, https://publications.europa.eu/en/publication-detail/-/ publication/dd711757-033f-11e9-adde-01aa75ed71a1/language-en. 